\title{
REMARKS ON AUTOMORPHISMS OF SUBFACTORS
}

\author{
PHAN H. LOI
}

(Communicated by Palle E. T. Jorgensen)

\begin{abstract}
We establish certain properties of automorphisms on an inclusion of AFD type $\mathrm{II}_{1}$ factors with finite index and finite depth and discuss their applications to the classification problem of AFD type III subfactors, including a different proof of a result on subfactors with principal graph $\tilde{D}_{n}$.
\end{abstract}

\section{INTRODUCTION}

It is a well-known fact that automorphisms play an important role in the study of von Neumann algebras as exemplified by their usefulness in the classification of AFD factors in $[2,3]$.

The importance of automorphisms of subfactors, however, has begun to emerge only recently, as the results $[19,13]$ show that automorphisms are also useful in the classification of certain AFD type III $_{\lambda}$ subfactors for $\lambda \in(0,1]$.

Recall that, for an inclusion $N \subset M$ of III $_{\lambda}$ factors, $\lambda \neq 0$, possessing a common discrete or continuous decomposition, there are two associated towers of higher relative commutants, one coming from $N \subset M$ and the other from the corresponding type II inclusion. The former tower is always embedded in the second one and can be realized as the pair of simultaneous fixed-point algebras of a certain class of automorphisms acting on the second tower (cf. [13]).

In general, these two towers alone do not entirely determine the conjugacy class of the type III subfactor. In this note we show that if we fix the type III and the type II towers, then there can only be finitely many AFD subfactors with identical derived towers in the type $\mathrm{III}_{\lambda}$ case, whereas there is either a unique or an uncountable number of such subfactors in the III $_{1}$ case.

In [6] Izumi and Kawahigashi have shown that the number of nonconjugate AFD type $\mathrm{II}_{1}$ subfactors with $\tilde{D}_{n}, n \geq 4$, as the principal graph is $n-2$ by computing explicitly the connection introduced by Ocneanu in [15]. These subfactors are in a one-to-one correspondence to the elements of a certain third cohomology group according to a result of Popa in [18]. We will give a proof of this result using automorphisms.

Received by the editors September 28, 1992.

1991 Mathematics Subject Classification. Primary 46L10, 46L55.

Key words and phrases. Subfactors, automorphisms.

The author was supported in part by a Research Challenge Grant at Wright State University. 


\section{Main Results}

Let $B \subset A$ be factors and $\operatorname{Aut}(A, B)=\{\alpha \in \operatorname{Aut}(A) ; \alpha(B)=B\} ; \alpha$ and $\beta$ are said to be outer conjugate in $\operatorname{Aut}(A, B)$ if they are conjugate in $\operatorname{Aut}(A, B)$ modulo $\operatorname{Int}(A, B)$, the group of inner automorphisms with unitaries from $B$.

Let us also recall the construction of the standard homomorphisms on a pair of types $\mathrm{II}_{1}$ or $\mathrm{II}_{\infty}$ factors with finite index. Let $B \subset A$ be such a pair, and let $\left\{B_{j}\right\}$ be a tunnel with Jones projections $e_{-j}$. Then there is a finite normal faithful trace defined on the tower of finite-dimensional algebras: $B_{j}^{\prime} \cap B \subset B_{j}^{\prime} \cap A$. Let $\mathscr{G}$ be the group of filtered automorphisms $\left\{\alpha_{j}\right\}$ which satisfy:

(1) $\alpha_{j}$ is an automorphism on $B_{j}^{\prime} \cap B \subset B_{j}^{\prime} \cap A$;

(2) $\alpha_{j}$ extends $\alpha_{j-1}$;

(3) $\alpha_{j}$ is trace-preserving for each $j$;

(4) $\alpha_{j}\left(e_{-k}\right)=e_{-k}$ for $0 \leq k \leq j-1$.

As a group, $\mathscr{G}$ is independent of the choice of the tunnel, and there exists a continuous homomorphism $\Phi: \operatorname{Aut}(A, B) \rightarrow \mathscr{G}$, called the standard homomorphism, such that $\operatorname{Ker}(\Phi)=\overline{\operatorname{Int}}(A, B)$ (cf. $[9,13])$.

Our first observation is that, for finite depth inclusions, the corresponding $\mathscr{G}$ cannot be too big.

Proposition 2.1. Let $B \subset A$ and $\mathscr{G}$ be as above. If $B \subset A$ has finite depth, then $\mathscr{G}$ is compact.

Proof. Since $B \subset A$ has finite depth, there is a $k_{0} \geq 0$ such that, for all $j \geq k_{0}, B_{j+1}^{\prime} \cap A=\left\{B_{j}^{\prime} \cap A, e_{-j}\right\}^{\prime \prime}$. It follows that an element $\alpha=\left\{\alpha_{j}\right\}$ of $\mathscr{G}$ is determined by $\alpha_{k_{0}}$, and the compactness of $\mathscr{G}$ follows from the finite dimensionality of $B_{k_{0}}^{\prime} \cap A$. Q.E.D.

The next lemma is an improved version of Lemma 4.2 of [14]; it relates the derived tower of $B \subset A$ to that of the pair of fixed-point algebras under a standard automorphism.

Lemma 2.2. Let $B \subset A$ be a pair of $A F D$ type $\mathrm{II}_{1}$ factors with finite index and finite depth. Let $\alpha$ be a standard automorphism of $B \subset A$ with respect to a generating tunnel. Then $\alpha$ has finite order if and only if $B^{\alpha} \subset A^{\alpha}$ has finite depth, and in this case the derived tower of $B^{\alpha} \subset A^{\alpha}$ can be computed as $\left(B_{k}^{\alpha}\right)^{\prime} \cap A^{\alpha}=\left(B_{k}^{\prime} \cap A\right)^{\alpha}$.

Proof. Suppose $B^{\alpha} \subset A^{\alpha}$ has finite depth. First we observe that because $B \subset A$ has finite depth by assumption and $\alpha$ is invariant with respect to the conditional expectations, $E_{B_{k}^{\prime} \cap A}, e_{-k}\left(B_{k}^{\prime} \cap A^{\alpha}\right) e_{-k}=\left(B_{k-1}^{\prime} \cap A^{\alpha}\right) e_{-k}$, for sufficiently large $k$. Fix such a $k$. Then it can be easily checked that

$$
\begin{array}{ccc}
\left\{B_{k}^{\prime} \cap A^{\alpha}, e_{-k}\right\}^{\prime \prime} & \subset & \left(B_{k+1}^{\alpha}\right)^{\prime} \cap A^{\alpha} \\
\cup & & \cup \\
B_{k}^{\prime} \cap A^{\alpha} & \subset & \left(B_{k}^{\alpha}\right)^{\prime} \cap A^{\alpha}
\end{array}
$$

is a commuting square. Performing the basic construction for $\left(B_{k}^{\alpha}\right)^{\prime} \cap A^{\alpha} \subset$ $\left(B_{k+1}^{\alpha}\right)^{\prime} \cap A^{\alpha}$, Lemma 4.6.3 in [4] implies that we have a sequence of commuting 
squares:

$$
\begin{array}{ccc}
\left\{B_{k}^{\prime} \cap A^{\alpha}, e_{-k}, \ldots, e_{-(k+l)}\right\}^{\prime \prime} & \subset & \left(B_{k+l+1}^{\alpha}\right)^{\prime} \cap A^{\alpha} \\
\cup & & \cup \\
\left\{B_{k}^{\prime} \cap A^{\alpha}, e_{-k}, \ldots, e_{-(k+l-1)}\right\}^{\prime \prime} & \subset & \left(B_{k+l}^{\alpha}\right)^{\prime} \cap A^{\alpha}
\end{array}
$$

Moreover, as $B^{\alpha} \subset A^{\alpha}$ has finite depth, the sequence of finite-dimensional algebras, $\left\{B_{k}^{\prime} \cap A^{\alpha}, e_{-k}, \ldots, e_{-(k+l-1)}\right\}^{\prime \prime} \subset\left\{B_{k}^{\prime} \cap A^{\alpha}, e_{-k}, \ldots, e_{-(k+l)}\right\}^{\prime \prime}$, is periodic. Hence since $\left\{B_{k}^{\prime} \cap A^{\alpha}, e_{-k}, \ldots, e_{-(k+l-1)}\right\}^{\prime \prime} \subset B_{k+l}^{\prime} \cap A^{\alpha} \subset\left(B_{k+l}^{\alpha}\right)^{\prime} \cap$ $A^{\alpha}$, it follows that the sequence $\left\{B_{k+l}^{\prime} \cap A^{\alpha}\right\}$ for $l \geq 1$ is also periodic, so we actually have $B_{k+l+1}^{\prime} \cap A^{\alpha}=\left\{B_{k+l}^{\prime} \cap A^{\alpha}, e_{-(k+l)}\right\}^{\prime \prime}$ for large $l$. Thus $B_{l}^{\prime} \cap A^{\alpha} \subset$ $\left(B_{l}^{\alpha}\right)^{\prime} \cap A^{\alpha}$ gives rise to a commuting square for sufficiently large $l$. As both $B_{l}^{\prime} \cap A^{\alpha}$ and $\left(B_{l}^{\alpha}\right)^{\prime} \cap A^{\alpha}$ approximate $A^{\alpha}$, we get that $B_{l}^{\prime} \cap A^{\alpha}=\left(B_{l}^{\alpha}\right)^{\prime} \cap A^{\alpha}$ for large $l$, so the two families of finite-dimensional algebras have the same principal graph. Therefore, $B_{l}^{\prime} \cap A^{\alpha}=\left(B_{l}^{\alpha}\right)^{\prime} \cap A^{\alpha}$ for all $l$. Now the commuting squares $B_{l}^{\prime} \cap A^{\alpha} \subset B_{l}^{\prime} \subset A$ satisfy the periodicity conditions in [21], and the two sequences approximate $A^{\alpha} \subset A$. Thus by the Wenzl index formula, [ $A$ : $\left.A^{\alpha}\right]<\infty$, so $\alpha$ has finite order.

For the converse, we refer the reader to the arguments in Lemma 4.2 of [14]. Q.E.D.

Proposition 2.3. Let $N \subset M$ be a pair of AFD type $\mathrm{III}_{\lambda}$ factors, $0<\lambda<1$, with finite index, finite depth, and a common discrete decomposition, and let $B \subset A$ be the corresponding type $\mathrm{II}_{1}$ pair. Then there are only finitely many nonconjugate pairs of AFD type $\mathrm{III}_{\lambda}$ factors with the same type II and type III towers as $N \subset M$.

Proof. By assumption $(N \subset M) \cong\left(B \otimes R_{0,1} \times_{\theta} \mathbf{Z} \subset A \otimes R_{0,1} \times_{\theta} \mathbf{Z}\right)$, where $R_{0,1}$ is the AFD type $\mathrm{II}_{\infty}$ factor and $\bmod (\theta)=\lambda$. By [13], $B \subset A$ has finite depth, so applying Theorem 4.2.1 in [19], we can assume that $\theta$ is of the form $\alpha \otimes \sigma$, where $\bmod (\sigma)=\lambda$ and $\alpha$ is a standard automorphism on $B \subset A$ with respect to some generating tunnel $B_{k}$. By [13], $N_{k}=\left(B_{k} \otimes R_{0,1}\right) \times_{\alpha \otimes \sigma} \mathbf{Z}$ gives rise to a tunnel of $N \subset M$ and $N_{k}^{\prime} \cap M=\left(B_{k}^{\prime} \cap A\right)^{\alpha}$; also the two tunnels share the same Jones projections. The finite depth condition of $N \subset M$ then implies that the sequence $\left\{B_{k}^{\prime} \cap A^{\alpha}\right\}$ is periodic. Since $B_{k}^{\prime} \cap A^{\alpha} \uparrow A^{\alpha}, A^{\alpha}$ is a type $\mathrm{II}_{1}$ factor; likewise so is $B^{\alpha}$. Using Lemma 2.2, we deduce that $\alpha$ has finite order, say $m$.

For any other AFD type III $_{\lambda}$ pair $Q \subset P$ satisfying the same hypotheses and having the same type II and type III towers as $N \subset M$, by the same argument as above, there is a standard automorphism $\beta$ with period $m$ on $B \subset A$ such that $B_{k}^{\prime} \cap A^{\alpha} \subset B_{k}^{\prime} \cap A$ is isomorphic to $B_{k}^{\prime} \cap A^{\beta} \subset B_{k}^{\prime} \cap A$. It follows that

$$
\begin{array}{ccccccc}
A^{\alpha} & \subset & A & & A^{\beta} & \subset & A \\
\cup & & \cup & \text { is isomorphic to } & \cup & & \cup, \\
B^{\alpha} & \subset & B
\end{array} \quad \begin{array}{lll}
B^{\beta} & \subset & B
\end{array}
$$

so $\beta$ is conjugate to some power $\alpha^{l}$ with $(m, l)=1$. By Theorem 4.2.1 in [19], $Q \subset P$ is classified by the conjugacy class of $\beta$ in the group of standard automorphisms on $B \subset A$; thus there can only be finitely many nonconjugate pairs. Q.E.D. 
Remark 2.4. In [14] it was shown that there exist uncountably many nonconjugate type III $_{\lambda}$ inclusions with $A_{\infty, \infty}$ as the type III principal graph and $\tilde{A}_{n}$ as the type II graph for any $n \geq 1$. Thus the finite depth condition in 2.3 is necessary.

The type $\mathrm{III}_{1}$ case, however, as we will see, is quite different. We recall that any type III $_{1}$ inclusion of factors admits a common continuous decomposition [12]. The next proposition has also been obtained independently by Popa in [19] and in the case where the index is less than 4 by Kosaki and Longo in [11].

Proposition 2.5. Let $N \subset M$ be a pair of type III $_{1}$ factors with minimal index, finite depth, and a common continuous decomposition $Q \times{ }_{\theta} \mathbf{R} \subset P \times_{\theta} \mathbf{R}$. Then $\Phi\left(\theta_{t}\right)=\mathbf{I d} \forall t \in \mathbf{R}$, where $\boldsymbol{\Phi}$ is the standard homomorphism.

Proof. Since the index is minimal (cf. [5]), by the arguments in [14], there is a tunnel $Q_{k}$ of $Q \subset P$ such that the derived tower of $N \subset M$ is isomorphic to $\left(Q_{k}^{\prime} \cap P\right)^{\Phi(\theta)}$. As $N \subset M$ has finite depth, Lemma 2.2 implies that $\Phi\left(\theta_{t}\right)=$ Id except for possibly finitely many $t$ 's. On the other hand, by the continuity of the standard homomorphism [14, 9], $\operatorname{Ker}(\Phi(\theta))$ is a closed subgroup of $\mathbf{R}$. Hence $\mathbf{R} / \operatorname{Ker}(\Phi(\theta))$ is finite, so we must have $\operatorname{Ker}(\Phi(\theta))=\mathbf{R}$, i.e., $\Phi\left(\theta_{t}\right)=$ Id, $\forall t$. Q.E.D.

By Theorem 4.2.1 in [19], any pair of AFD type III $_{1}$ factors with finite depth splits as a tensor product of a pair of AFD $\mathrm{II}_{1}$ factors with a common AFD type III $_{1}$ factor.

Proposition 2.6. Let $N \subset M$ be AFD type $\mathrm{III}_{1}$ factors with minimal index, and let $B \subset A$ be the corresponding pair of $A F D$ type $\mathrm{II}_{1}$ factors in the common continuous decomposition of $N \subset M$. Then either $N \subset M$ is isomorphic to $B \otimes R_{\infty} \subset A \otimes R_{\infty}$, where $R_{\infty}$ is the unique $A F D$ factor of type III $_{1}$, or there exist uncountably many nonconjugate AFD pairs of type $\mathrm{III}_{1}$ factors with the same type III and II towers as $N \subset M$.

Proof. Let $\theta_{t}, t \in \mathbf{R}$ denote the trace-scaling one-parameter goup that arises in the common continuous decomposition of $N \subset M$. Let $\Phi$ denote the standard homomorphism. If $\Phi\left(\theta_{t}\right)=$ Id $\forall t \in \mathbf{R}$, then $N \subset M \cong B \otimes R_{\infty} \subset A \otimes R_{\infty}$, by Theorem 4.2.1 of [19]. Otherwise $\operatorname{Ker}(\Phi(\theta))=t_{0} \mathbf{Z}$ for some $t_{0}$ which is nonzero by Lemma 2.2. Now for each $\varepsilon>0$, define $\theta_{t}^{\varepsilon}=\theta_{\varepsilon t}$ for $t \in \mathbf{R}$, then $\operatorname{Ker}\left(\Phi\left(\theta^{\varepsilon}\right)\right)=\left(t_{0} \varepsilon^{-1}\right) \mathbf{Z}$. Hence if $\varepsilon \neq \varepsilon^{\prime}$, then $\Phi\left(\theta^{\varepsilon}\right)$ and $\Phi\left(\theta^{\varepsilon^{\prime}}\right)$ are nonconjugate in $\mathscr{G}$, so $\theta^{\varepsilon}$ and $\theta^{\varepsilon^{\prime}}$ are not outer conjugate on $B \otimes R_{0,1} \subset$ $A \otimes R_{0,1}$, because $\Phi$ maps outer conjugacy into conjugacy. Thus the resulting pair of type III $_{1}$ factors by taking the crossed products of $B \otimes R_{0,1} \subset A \otimes R_{0,1}$ with $\theta^{\varepsilon}$ are pairwise nonconjugate; yet their type III towers are all equal to that of $N \subset M$, since $\left(B_{k}^{\prime} \cap A\right)^{\Phi(\theta)}=\left(B_{k}^{\prime} \cap A\right)^{\Phi\left(\theta^{e}\right)}$. Q.E.D.

As the preceding propositions indicate, for an inclusion of AFD type III $_{\lambda}$ factors with $\lambda \in(0,1]$, the relation between the type III and type II towers is quite interesting. It is in the course of computing these towers explicitly in the index $=4$ case that we realize that a proof of a result of Izumi and Kawahigashi in [6] on the number of nonconjugate AFD type $\mathrm{II}_{1}$ subfactors of $R$ based on automorphisms can be obtained. According to [18], such subfactors are in a one-to-one correspondence with the elements of a certain third cohomology 
group. The proof in [6] that there are $n-2$ nonconjugate pairs of AFD type $\mathrm{II}_{1}$ factors with the Coxeter-Dynkin diagram $\tilde{D}_{n}$ as the principal graph is based on an explicit calculation of the connection of the paragroup, which was introduced in [15], corresponding to such a pair of factors. Thus it might be of some interest that the automorphism approach is also useful in the classification problem for subfactors.

Before we get into the details of the proof, we need to recall certain facts from [14].

Let $N \subset M$ be a pair of AFD type $\mathrm{II}_{1}$ factors with $\tilde{A}_{n}, n \geq 2$, as principal graph. Note that in this paper $\tilde{A}_{n}$ has $2 n$ vertices. Then there exists a period 2 outer automorphism $\alpha$ on $N \subset M$, which is standard with respect to some generating tunnel and such that the pair of fixed-point algebras $N^{\alpha} \subset M^{\alpha}$ are type $\mathrm{II}_{1}$ factors with $\tilde{D}_{n+2}$ as the principal graph. By Takesaki duality, there exists a period 2 outer automorphism $\beta$ on $B \subset A$ such that $\left(B \times{ }_{\beta} \mathbf{Z}_{2} \subset\right.$ $\left.A \times{ }_{\beta} \mathbf{Z}_{2}\right) \cong(N \subset M)$.

The idea of our proof consists of showing that $\alpha$ and $\beta$ with the properties above are unique up to outer conjugacy on the respective inclusion of factors.

We begin with a simple lemma. In the following, all factors are assumed to be AFD of type $\mathrm{II}_{1}$.

Lemma 2.7. Let $B \subset A$ be factors with finite index and finite depth. Let $\alpha$ be a nontrivial standard automorphism on $B \subset A$ with respect to a generating tunnel $\left\{B_{k}\right\}$. Then $\alpha$ is outer on each of the factors, $\cdots \subset B_{k} \subset \cdots \subset B \subset A$.

Proof. Clearly $\alpha$ is outer on $B$ because it is standard and nontrivial-similarly on $B_{k}$ for each $k$. If $\alpha=\operatorname{Ad} u$, for some $u \in M$, then $\alpha^{l}$ must be inner on $B$ for some $l$ and hence is trivial. Hence $\alpha$ has finite order, so $B^{\alpha}$ and $B_{1}^{\alpha}$ are factors. But then $A^{\alpha}$ is the basic extension of $B_{1}^{\alpha} \subset B^{\alpha}$ and hence is a factor and $u$ is a scalar, so $\alpha=\mathrm{Id}$, a contradiction. Hence $\alpha$ must be outer on $A$. Q.E.D.

The following notion of strongly or properly outer actions on a pair of factors $B \subset A$ was introduced in [1,20]: $\alpha \in \operatorname{Aut}(A, B)$ is called strongly or properly outer on $B \subset A$ if there is no nonzero $\alpha \in A$ such that $a \alpha(x)=x \alpha$, for $x \in B$.

The opposite notion to strongly outerness is that of weakly innerness; namely, $\alpha \in \operatorname{Aut}(A, B)$ is called weakly inner on $B \subset A$ if there is a nonzero $\alpha \in A$ such that $a \alpha(x)=x a$, for all $x \in B$. Note that if $B^{\prime} \cap A=\mathbf{C}$, then this condition means that there is a unitary $u \in A$ such that $\alpha=\operatorname{Ad} u$ on $B$. By Proposition 7 in [1], if $\left(B^{\prime} \cap A\right)^{\alpha} \neq(B \times \alpha)^{\prime} \cap(A \times \alpha)$, then $\alpha$ is weakly inner on $B \subset A$.

Proposition 2.8. Let $\alpha$ be a standard automorphism with finite order on $B \subset A$ with respect to a generating tunnel $\left\{B_{k}\right\}$. Then the predual $\beta$ of $\alpha$ is weakly inner on $B_{k}^{\alpha} \subset A^{\alpha}$ for some $k$ less than or equal to the depth of $B \subset A$.

Proof. We have $\left(B_{k}^{\alpha} \times{ }_{\beta} \mathbf{Z}_{n} \subset A^{\alpha} \times{ }_{\beta} \mathbf{Z}_{n}\right) \cong\left(B_{k} \subset A\right)$, for all $k$. Hence $\left(B_{k}^{\alpha} \times{ }_{\beta} \mathbf{Z}_{n}\right)^{\prime} \cap\left(A^{\alpha} \times{ }_{\beta} \mathbf{Z}_{n}\right) \cong B_{k}^{\prime} \cap A$. On the other hand, $\left(\left(B_{k}^{\alpha}\right)^{\prime} \cap A^{\alpha}\right)^{\beta} \subset$ $\left(B_{k}^{\alpha}\right)^{\prime} \cap A^{\alpha}=\left(B_{k} \cap A\right)^{\alpha}$, which is strictly contained in $B_{k}^{\prime} \cap A$ for some $k$ not exceeding the depth of $B \subset A$ because $\alpha$ is nontrivial. Therefore, $\beta$ is weakly inner on $B_{k} \subset A$. Q.E.D. 
Theorem 2.9. Let $B \subset A$ be a pair of AFD type $\mathrm{II}_{1}$ factors with principal graph $\tilde{D}_{n}, n \geq 4$ (resp. $\left.D_{\infty}\right)$, and $\beta$ a period 2 outer automorphism on $B \subset A$ such that $B \times{ }_{\beta} \mathbf{Z}_{2} \subset A \times{ }_{\beta} \mathbf{Z}_{2}$ has principal graph $\tilde{A}_{n-2}$ (resp. $\left.A_{\infty, \infty}\right)$. Then $\beta$ is unique up to outer conjugacy.

Proof. Since $B^{\prime} \cap A=\mathbf{C}$, by the remark preceding Proposition 2.8, $\beta$ is weakly inner on $B \subset A$. Let us consider first the cases of the $\tilde{D}_{n}$ graph for $n \geq 5$ or the $D_{\infty}$ graph. It is then well known that in these cases the normalizer $\mathscr{N}(B)$ of $B$ in $A$ generates a subfactor of index 2 in $A$. By Goldman's theorem in [8], there exist period 2 outer automorphisms $\sigma$ and $\rho$ such that $\mathscr{N}(B)^{\prime \prime}=B \times_{\sigma} \mathbf{Z}_{2}$ and $A=\mathscr{N}(B)^{\prime \prime} \times_{\rho} \mathbf{Z}_{2}$. From now on $\sigma$ and $\rho$ are fixed, and we let $u_{\sigma}$ and $u_{\rho}$ be the corresponding implementing unitaries. As $\beta$ is weakly inner on $B \subset A$, $\beta \mid B$ is implemented by a unitary in $\mathscr{N}(B)$ which is necessarily of the form $w u_{\sigma}$. Hence by perturbing $\beta$ with $\operatorname{Ad} w$, we may assume that $\beta \mid B=\operatorname{Ad} u_{\sigma}$. Using Galois Theory for finite group actions, we see that on $\mathscr{N}(B)^{\prime \prime}=B \times_{\sigma} \mathbf{Z}_{2}$ either $\beta=\operatorname{Ad} u_{\sigma}$ or $\beta=\operatorname{Ad} u_{\sigma} \hat{\sigma}$. We claim that the second possibility cannot occur, for if $\beta=\operatorname{Ad} u_{\sigma} \hat{\sigma}$, then $\hat{\sigma}$ can be extended to an outer automorphism of $A$ whose fixed-point algebra is a subfactor of index 2 of $A$ and which contains $B$. Hence it must be equal to $\mathscr{N}(B)^{\prime \prime}=B \times{ }_{\sigma} \mathbf{Z}_{2}$. This implies that $\hat{\sigma}=$ Id on $B \times_{\sigma} \mathbf{Z}_{2}$, a contradiction! Therefore, $\beta=\operatorname{Ad} u_{\sigma}$ on $B \times_{\sigma} \mathbf{Z}_{2}$. Repeating the same argument as above, we deduce that $\beta=\operatorname{Ad} u_{\sigma} \hat{\rho}$ on $A$. Since $\sigma$ and $\rho$ are fixed throughout, $\beta$ is actually unique up to inner perturbation by a unitary in $B$.

If the principal graph is $\tilde{D}_{4}$, then either $A=B \times_{\theta} \mathbf{Z}_{4}$ or $A=B \times_{\theta}\left(\mathbf{Z}_{2} \oplus \mathbf{Z}_{2}\right)$ for some outer action $\theta$.

In the first case, the same argument as above shows that up to an inner perturbation by a unitary in $B, \beta=\operatorname{Ad} u_{\theta} \hat{\theta}^{2}$, so it is unique.

In the second case, set $G=\mathbf{Z}_{2} \oplus \mathbf{Z}_{2}$, and let $u_{i}, i \in G$, be the implementing unitaries of $\theta$. Then as above $\beta=\operatorname{Ad} u_{i} \hat{\theta}_{j}$ for some $i \in G$ and $\hat{\jmath} \in \hat{G}$. We will show that all automorphisms on $B \subset A$ having these forms are outer conjugate to each other. First, by the classification up to conjugacy of finite group actions on the AFD type $\mathrm{II}_{1}$ factor in [7], for any $i, j \in G$, there is an automorphism $\varphi$ on $B$ such that $\varphi \theta_{i} \varphi^{-1}=\theta_{j}$. Using standard arguments, $\varphi$ can then be extended to an automorphism on $B \times{ }_{\theta} G$ which intertwines the dual action $\hat{\theta}$. It follows that $\varphi\left(\operatorname{Ad} u_{i} \hat{\theta}_{k}\right) \varphi^{-1}=\operatorname{Ad} u_{j} \hat{\theta}_{k}$, for any $i, j \in G$ and any $\hat{k} \in \hat{G}$. Now it remains to show that $\operatorname{Ad} u_{i} \hat{\theta}_{j}$ is outer conjugate to $\operatorname{Ad} u_{j} \hat{\theta}_{k}$ for some $i, j \in G$ and any $\hat{\jmath}, \hat{k} \in \hat{G}$, with $\hat{\jmath} \neq \hat{k}$. Again, by the classification of finite group actions on $R$ in [7], there exists an automorphism $\varphi$ on $B \times{ }_{\theta} G$ which permutes the dual action in such a way that $\varphi \hat{\theta}_{j} \varphi^{-1}=\hat{\theta}_{\hat{k}}$. Since $\varphi$ must preserve the fixed-point algebra of the dual action which is $B, \varphi\left(u_{i}\right)=w u_{j}$, for some $j \in G$ and some unitary $w$ in $B$. Hence $\left(\operatorname{Ad} w^{*} \varphi\right) \operatorname{Ad} u_{i}=\operatorname{Ad} u_{j} \varphi^{-1}$ and we have $\left(\operatorname{Ad} w^{*} \varphi\right)\left(\operatorname{Ad} u_{i} \hat{\theta}_{\hat{j}}\right)=\operatorname{Ad} u_{j}\left(\varphi \hat{\theta}_{j}\right)=\left(\operatorname{Ad} u_{j} \hat{\theta}_{\hat{k}}\right) \varphi$. Thus $\operatorname{Ad} u_{i} \hat{\theta}_{\hat{j}}$ is outer conjugate to $\operatorname{Ad} u_{j} \hat{\theta}_{\hat{k}}$. Q.E.D.

Theorem 2.10. Let $N \subset M$ be a pair of AFD type $\mathrm{II}_{1}$ factors with principal graph $\tilde{A}_{n}$ or $A_{\infty, \infty}$, and let $\alpha \in \operatorname{Aut}(M, N)$ be an outer automorphism with period 2 such that $\left(N^{\alpha}\right)^{\prime} \cap M^{\alpha}=\mathrm{C}$. Then $\alpha$ is unique up to outer conjugacy.

Proof. By a result in [17], $N \subset M$ can be identified with the inclusion $\{x \oplus$ 
$\theta(x) ; x \in R\} \subset R \otimes M_{2}(\mathbf{C})$, where $\theta$ is an automorphism of the AFD type $\mathrm{II}_{1}$ factor $R$ which has outer period $n$ if the principal graph of $N \subset M$ is $\tilde{A}_{n}$ or is aperiodic if the graph is $A_{\infty, \infty}$.

Let $\varepsilon_{i j}$ be the matrix units in the identification above of $M$ so that $N^{\prime} \cap$ $M=\mathrm{C} e_{11} \oplus \mathrm{Ce}_{22}$. Let $\alpha$ be an automorphism satisfying the hypotheses in the theorem. Then as $N^{\alpha}$ has a trivial relative commutant in $M^{\alpha}, \alpha\left(e_{11}\right)=e_{22}$.

A simple calculation reveals that $\alpha$ determines a unique automorphism $\tilde{\alpha}$ on $R$ and a unique unitary $c$ in $R$ such that

$$
\alpha\left(e_{12}\right)=c e_{21} \quad \text { and } \quad \alpha=\operatorname{Ad}\left[\begin{array}{ll}
0 & 1 \\
c & 0
\end{array}\right](\tilde{\alpha} \otimes \mathrm{Id}) .
$$

We observe that in $M$ the projections $e=\frac{1}{2}\left[\begin{array}{ll}1 & 1 \\ 1 & 1\end{array}\right]$ and $f=\frac{1}{2}\left[\begin{array}{ll}1 & c^{*} \\ c\end{array}\right]$ have the same trace which is equal to the inverse of the index. Thus by [17] there is a unitary $u$ in $R$ such that $\operatorname{Ad}\left[\begin{array}{ll}u & 0 \\ \theta(u)\end{array}\right] e=f$. From this it follows that $c=\theta(u) u^{*}$ and hence

$$
\operatorname{Ad}\left[\begin{array}{cc}
u^{*} & 0 \\
0 & \theta\left(u^{*}\right)
\end{array}\right] \alpha=\operatorname{Ad}\left[\begin{array}{ll}
0 & 1 \\
1 & 0
\end{array}\right]\left(\operatorname{Ad} u^{*} \tilde{\alpha} \otimes \operatorname{Id}\right) .
$$

The fact that $\alpha$ preserves $N$ entails that $\left(\operatorname{Ad} u^{*} \tilde{\alpha}\right) \theta\left(\operatorname{Ad} u^{*} \tilde{\alpha}\right)^{-1}=\theta^{-1}$. Since we are interested in the outer conjugacy of $\alpha$, we may assume that $\alpha$ has the form of the right-hand side in the equality above.

Suppose that $\alpha^{\prime}$ is another automorphism on $N \subset M$ satisfying the same conditions as $\alpha$; we let $\tilde{\alpha}^{\prime}, u^{\prime}$ be defined similarly as above.

Now $\left\{\theta, \operatorname{Ad} u^{*} \tilde{\alpha}\right\}$ and $\left\{\theta, \operatorname{Ad} u^{\prime *} \tilde{\alpha}^{\prime}\right\}$ determine two actions of the semidirect product $\mathbf{Z} \times{ }_{\sigma} \mathbf{Z}_{2}$ ( $\sigma$ denotes the inversion symmetry on $\mathbf{Z}$ ) on $R$ which have the same outer invariant by a straightforward computation. By the classification result of countable discrete amenable group actions on $R$ in [16], these two actions are outer conjugate in $R$, i.e., there is an automorphism $\varphi$ of $R$ and a $\left(\mathbf{Z} \times{ }_{\sigma} \mathbf{Z}_{2}\right)$-cocycle $u_{(i, j)}$ such that $\operatorname{Ad} u_{(1,0)} \theta_{\varphi}=\varphi \theta$ and $\operatorname{Ad} u_{(0,1)}\left(\operatorname{Ad} u^{\prime *} \tilde{\alpha}^{\prime}\right) \varphi=\varphi\left(\operatorname{Ad} u^{*} \tilde{\alpha}\right)$.

It follows that

$$
\Phi=\operatorname{Ad}\left[\begin{array}{cc}
1 & 0 \\
0 & u_{(0,1)}^{*}
\end{array}\right](\varphi \otimes I d)
$$

defines an automorphism on $N \subset M$. We have

$$
\begin{aligned}
\Phi \alpha \Phi^{-1} & =\Phi\left(\operatorname{Ad}\left[\begin{array}{ll}
0 & 1 \\
1 & 0
\end{array}\right]\left(\operatorname{Ad} u^{*} \tilde{\alpha} \otimes \mathrm{Id}\right)\right) \Phi^{-1} \\
& =\operatorname{Ad}\left[\begin{array}{cc}
u_{(-1,1)} & 0 \\
0 & u_{(1,0)}^{*} u_{(0,1)}
\end{array}\right] \alpha^{\prime} .
\end{aligned}
$$

As $u_{(0,1)} \theta\left(u_{(-1,1)}\right)=u_{(0,1)}$,

$$
\left[\begin{array}{cc}
u_{(-1,1)} & 0 \\
0 & u_{(1,0)}^{*} u_{(0,1)}
\end{array}\right] \in N
$$

so $\alpha$ and $\alpha^{\prime}$ are outer conjugate in $N \subset M$. Q.E.D.

Let us now show that for each $n \geq 4$, there are $n-2$ nonconjugate subfactors of $R$ with $\tilde{D}_{n}$ as the principal graph.

First of all, it is known that, by [16], each subfactor of $R$ with principal graph $\tilde{A}_{n}, n \geq 2$, is locally trivial and so classified, up to isomorphism, by 
Connes's obstruction of the corresponding automorphism, which is an $n$th root of 1 (cf. [3]). Hence there are exactly $n$ nonconjugate such subfactors of $R$. By Theorem 4.4 in [14], each pair of AFD factors with principal graph $\tilde{A}_{n-2}$, $n \geq 4$, contains a pair of subfactors as the fixed-point algebras of a period 2 standard outer automorphism on $N \subset M$ and which has $\tilde{D}_{n}$ as the principal graph. By Theorem 2.9, these $\tilde{D}_{n}$ pairs are mutually nonconjugate; hence, there are at least $n-2$ of them.

Conversely, starting with a $\tilde{D}_{n}$ inclusion $B \subset A$, let $\beta$ be the weakly inner automorphism on $B \subset A$ that is given in Theorem 2.9. Then the pair of crossed product factors $N=B \times_{\beta} \mathbf{Z}_{2} \subset M=A \times_{\beta} \mathbf{Z}_{2}$ has index 4 and a nontrivial relative commutant. Based on the list in [4], the principal graph of $N \subset M$ is either $\tilde{A}_{m}$ or $A_{\infty, \infty}$. By Theorem $2.10, \tilde{\beta}$ is unique up to outer conjugacy on $N \subset M$ and there is a unique period 2 automorphism on the $A_{\infty, \infty}$ inclusion whose pair of fixed-point algebras has $D_{\infty}$ as the principal graph. Hence the possibility of the $A_{\infty, \infty}$ graph is ruled out, so the principal graph for $N \subset M$ is $\tilde{A}_{m}$ and the same argument shows that $m=n-2$. In other words, any $\tilde{D}_{n}$ subfactor of $R$ is conjugate to exactly one of the $n-2$ subfactors constructed as the pair of fixed-point algebras of a period 2 outer automorphism on an $\tilde{A}_{n-2}$ pair of factors.

Remark 2.11. (1) It should be noted that the arguments in Theorem 2.10 can also be made to work for more general automorphisms, not just for those with period 2.

(2) It follows from Theorem 2.10 that the automorphism constructed in [9] based on the orbifold method is outer conjugate to the standard one constructed in [14].

\section{REFERENCES}

1. M. Choda and H. Kosaki, Strongly outer actions for an inclusion of factors, preprint, 1992.

2. A. Connes, Classification of injective factors, Ann. of Math. (2) 104 (1976), 73-115.

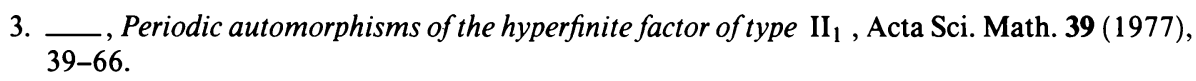

4. F. Goodman, P. de la Harpe, and V. F. R. Jones, Coxeter graphs and towers of algebras, Publ. Math. Sci. Res. Inst. 14 (1989).

5. F. Hiai, Minimizing indices of conditional expectations onto a subfactor, Publ. Res. Inst. Math. Sci. 24 (1988), 673-678.

6. M. Izumi and Y. Kawashigashi, Classification of subfactors with the principal graph $D_{n}^{(1)}$, Res. Inst. Math. Sci., preprint, 1991.

7. V. F. R. Jones, Actions of finite groups on the hyperfinite type $\mathrm{II}_{1}$ factor, Mem. Amer. Math. Soc., vol. 28, Amer. Math. Soc., Providence, RI, 1980.

8. __ Index for subfactors, Invent. Math. 72 (1983), 1-25.

9. Y. Kawahigashi, Automorphisms commuting with a conditional expectation onto a subfactor with finite index, J. Operator Theory (to appear).

10. _ On flatness of Ocneanu's connections on the Dynkin diagrams and classification of subfactors, Univ. of Tokyo, preprint, 1991.

11. H. Kosaki and R. Longo, A remark on the minimal index for subfactors, preprint, 1991.

12. P. H. Loi, On the theory of index and type III factors, Thesis, Penn. State Univ., 1988.

13. __ On automorphisms of subfactors, preprint, 1990. 
14. _ On the derived towers of certain inclusions of type $\mathrm{III}_{\lambda}$ factors of index 4 , Pacific $\mathrm{J}$. Math. (to appear).

15. A. Oceanu, Quantized groups, string algebras and Galois theory for algebras, Operator Algebras and Applications, Vol. 2, London Math. Soc. Lecture Note Ser., vol. 136, Cambridge Univ. Press, Cambridge and New York, 1988, pp. 119-182.

16. __ Actions of discrete amenable groups on the hyperfinite type $\mathrm{II}_{1}$ factor, Lecture Notes in Math., vol. 1138, Springer-Verlag, Berlin and New York, 1986.

17. M. Pimsner and S. Popa, Entropy and index for subfactors, Ann. Sci. École Norm. Sup. (4) 19 (1986), 57-106.

18. S. Popa, Sur la classification des sousfacteurs d'indice fini dans un facteur hyperfini, C. R. Acad. Sci. Paris Sér. I. Math. 311 (1990), 95-100.

19. __ Classification of amenable subfactors and their automorphisms, preprint, 1991.

20. _ On the classification of actions of amenable groups on factors, C. R. Acad. Sci. Paris Sér. I. Math. (to appear).

21. H. Wenzl, Hecke algebras of type $A$ and subfactors, Invent. Math. 92 (1988), 345-383.

Department of Mathematics and Statistics, Wright State University, Dayton, Ohio 45435

E-mail address: ploi@desire.wright.edu 